\title{
Selective cytotoxicity of green synthesized silver nanoparticles against the MCF-7 tumor cell line and their enhanced antioxidant and antimicrobial properties
}

This article was published in the following Dove Press journal:

International Journal of Nanomedicine

\author{
Sadegh Khorrami' \\ Ali Zarrabi' \\ Moj Khaleghi \\ Marziyeh Danaei ${ }^{3}$ \\ MR Mozafari ${ }^{3}$
}

'Department of Biotechnology, Faculty of Advanced Sciences and Technologies, University of Isfahan, Isfahan, Iran; ${ }^{2}$ Department of Biology, Faculty of Sciences, Shahid Bahonar University, Kerman, Iran; ${ }^{3}$ Australasian Nanoscience and Nanotechnology Initiative, Monash University, Clayton, VIC, Australia
Correspondence: Ali Zarrabi Department of Biotechnology, Faculty of Advanced Sciences and Technologies, University of Isfahan, Hezar Jarib St, 8I7467344I, Isfahan, Iran

$\mathrm{Tel}+983137934360$

Fax +9831 37932342

Email a.zarrabi@ast.ui.ac.ir
Introduction: Silver nanoparticles (AgNPs) are of great interest due to their unique and controllable characteristics. Different synthesis methods have been proposed to produce these nanoparticles, which often require elevated temperatures/pressures or toxic solvents. Thus, green synthesis could be a replacement option as a simple, economically viable and environmentally friendly alternative approach for the synthesis of silver nanoparticles.

Methods: Here, the potential of the walnut green husk was investigated in the production of silver nanoparticles. An aqueous solution extracted from walnut green husk was used as a reducing agent as well as a stabilizing agent. Then, the synthesized nanoparticles were characterized with respect of their anticancer, antioxidant, and antimicrobial properties.

Results: Results showed that the synthesized nanoparticles possessed an average size of 31.4 $\mathrm{nm}$ with a Zeta potential of $-33.8 \mathrm{mV}$, indicating high stability. A significant improvement in the cytotoxicity and antioxidant characteristics of the green synthesized Ag nanoparticles against a cancerous cell line was observed in comparison with the walnut green husk extract and a commercial silver nanoparticle (CSN). This could be due to a synergistic effect of the synthesized silver nanoparticles and their biological coating. AgNPs and the extract exhibited $70 \%$ and $40 \%$ cytotoxicity against MCF-7 cancerous cells, respectively, while CSN caused 56\% cell death (at the concentration of $60 \mu \mathrm{g} / \mathrm{mL}$ ). It was observed that AgNPs were much less cytotoxic when tested against a noncancerous cell line (L-929) in comparison with the control material (CSN). The free radical scavenging analysis demonstrated profound anti-oxidant activity for the synthesized nanoparticles in comparison with the extract and CSN. It was also detected that the synthesized AgNPs possess antibacterial activity against nosocomial and standard strains of both Grampositive and Gram-negative bacteria (minimum inhibitory concentration $=5-30 \mu \mathrm{g} / \mathrm{mL}$ ).

Conclusion: These findings imply that the synthesized nanoparticles using green nanotechnology could be an ideal strategy to combat cancer and infectious diseases.

Keywords: green synthesis, silver nanoparticles, antimicrobial agent, antioxidant agent, anticancer agent, selective cytotoxicity

\section{Introduction}

Despite promising achievements in pharmaceutical biotechnology and development of new drugs, cancer and infectious diseases are still the main causes of mortality and morbidity in the world after cardiovascular diseases. ${ }^{1}$ Besides, most microorganisms are intrinsically capable of evading therapeutic agents due to their rapid evolutionary genetic mechanisms of adaptation. ${ }^{2}$ On the other hand, many pharmaceutical companies 
have lost their interest in developing new antibiotic compounds due to their narrow profit margin. ${ }^{3}$ Consequently, it is crucial to develop efficient and low-cost processes for the production of therapeutic agents to combat the mentioned health problems. Significant developments in nanoscience and nanotechnology, particularly in the area of diagnosis and treatment, are promising toward tackling the issue of the development of antimicrobial drug resistance and improving life quality. Biocompatible nanomaterial with engineered physicochemical properties can provide effective strategies for defeating cancer and infectious disease. ${ }^{4-9}$

Different physical and chemical methods have been proposed to produce biocompatible nanoparticles with enhanced therapeutic properties. The physical methods often require elevated temperatures and/or high pressures that may not be cost effective for large-scale manufacture of the nanoparticles. Nevertheless, certain chemical methods are capable of producing nanoparticles with desirable characteristics. Utilization of toxic and hazardous solvents, however, is one of the shortfalls of the chemical processes. ${ }^{10-14}$

Recently, green synthesis has been introduced as a simple, economically viable, and environmentally friendly alternative approach for the synthesis of nanoparticles. In a typical green synthesis, biological compounds (such as plant extracts), microorganisms, or even eukaryotic cells act as both reducing agent and stabilizing agent leading to the production of desirable nanoparticles with predefined features. ${ }^{11,15,16}$

Silver nanoparticles (AgNPs) are of great interest due to their unique and controllable characteristics. These include antioxidant, antifungal, anti-inflammatory, antiviral, antiangiogenesis, and antimicrobial effects. ${ }^{17-19}$ Furthermore, there are increasing reports on the antitumor properties of AgNPs. ${ }^{19-22}$ However, their mechanism of action against microorganisms and the cancerous cells as well as their toxicity effect on normal cells has not been yet fully understood.

In this study, we have investigated the feasibility of using an aqueous solution extracted from walnut green husk for the synthesis of AgNPs. A very mild and solvent-free procedure was employed at a low temperature for the extraction of the herbal material. We propose that this biosafe extract acts as a reducing agent as well as a stabilizing agent. The synthesized nanoparticles were assessed in terms of their cytotoxicity and characterized with respect of their antioxidant, antitumor, and antimicrobial properties.

\section{Materials and methods}

\section{Materials}

The walnut fruits (Juglans regia) were collected from the University of Isfahan campus, Isfahan, Iran. Silver nitrate
(99.98\%) was purchased from Merck (Darmstadt, Germany). 1,1-diphenyl-2-picrylhydrazyl (DPPH) was procured from Sigma-Aldrich (Darmstadt, Germany). Commercial silver nanoparticles (CSNs) (used as a control) were product of US Research Nanomaterials Inc. (Houston, TX, USA). High-purity chemical reagents were purchased from Merck and Aldrich chemical companies. All other materials were of commercial reagent grade. Bacterial strains of Escherichia coli, Pseudomonas aeruginosa, and Staphylococcus aureus were obtained from Isfahan University of Medical Sciences, Isfahan, Iran. L-929 and MCF-7 cell lines and standard strains of the same bacteria by standard numbers 35218 ATCC (American Type Culture Collection), 1214 PTCC (Persian Type Culture Collection), and 1189 ATCC, respectively, were purchased from Pasteur Institute of Iran.

\section{Preparation of walnut extract}

The walnuts were washed, peeled, and $10 \mathrm{~g}$ of their husks was mixed with $100 \mathrm{~mL}$ deionized water. The solution was agitated at $37^{\circ} \mathrm{C}$ for 24 hours in a shaker incubator. The extract was then filtered using Whatman No 1 filter papers. The filtrate was concentrated by rotary evaporator $\left(70^{\circ} \mathrm{C}\right)$ and then dried under lab conditions $\left(27^{\circ} \mathrm{C}-30^{\circ} \mathrm{C}\right)$ to make a powder form of the extract.

\section{Green synthesis of silver nanoparticles (AgNPs)}

AgNPs were synthesized using the following protocol: $50 \mathrm{mg}$ of the dried walnut extract was dissolved in $100 \mathrm{~mL}$ deionized water and its $\mathrm{pH}$ was adjusted to 8 by using $\mathrm{NaOH}$. Then, $100 \mathrm{~mL}$ of the silver nitrate solution $(6 \mathrm{mM})$ was gradually added to the extract solution while stirring (1,000 rpm). The flask containing the sample was stirred at $37^{\circ} \mathrm{C}-40^{\circ} \mathrm{C}$ in dark for 24 hours. The resultant colorful mixture (dark brown) was then centrifuged at $13,000 \mathrm{rpm}$ for 15 minutes at $25^{\circ} \mathrm{C}$. The obtained pellet was washed twice with deionized water to remove any residues of the extract. The final precipitate was lyophilized and stored for further characterization.

\section{Characterization of the AgNPs}

\section{UV-Vis spectroscopy}

The ultraviolet-visible (UV-Vis) spectroscopy (JASCO V-670 UV-VIS-NIR Spectrophotometer; Tokyo, Japan) was used within the range of 300-700 nm for assessing the effect of time on AgNP formation. Optical properties of the metal nanoparticles, which are sensitive to concentration, size, shape and agglomeration state, make UV-Vis spectroscopy a valuable tool for identifying these materials. The formation of unique peak at specific wavelength of light is due to the surface plasmon resonance of the electrons present on the nanoparticle surface. 


\section{Particle size and charge analysis}

The hydrodynamic size ( $\mathrm{Z}$ average), polydispersity index (PDI), and surface charge (zeta potential) of the synthesized nanoparticles were analyzed by the Horiba SZ-100 particle size analyzer (Kyoto, Japan). Particle size analysis was performed at the scattering angle of $90^{\circ}$, medium viscosity $0.895 \mathrm{mPa} \cdot \mathrm{s}$, count rate of $210 \mathrm{kCPS}$, at $25^{\circ} \mathrm{C}$.

\section{Morphological investigation}

The field emission scanning electron microscopy (FESEM) (MIRA3; TESCAN, Brno, Czech Republic) was employed for investigating the morphology of the AgNPs as well as presence/absence of any aggregation or agglomeration.

\section{X-ray diffraction analysis}

Crystallographic analyses of the AgNPs were performed using X-ray powder diffraction (XRD; XRD D8 Advance, Bruker, Madison, WI, USA) having $\mathrm{Cu} \mathrm{K} \alpha$ $(\lambda=1.540 \AA)$ as a radiation source within $2 \theta=10-700$ at the scan speed $0.4 \% \mathrm{~min}$. The obtained pattern from the XRD analysis was used to identify the chemical composition and crystalline structure of the compound. The chemical composition of nanoparticles was also assessed using energy-dispersive X-ray spectroscopy (EDS) (MIRA3; TESCAN).

\section{Fourier transform infrared spectroscopy}

In order to verify the possible effect of various phytochemicals present in the walnut green husk extract on the surface modification of the synthesized nanoparticles, Fourier transform infrared (FTIR) spectroscopy was employed (JASCO Ltd., Tokyo, Japan).

\section{Antioxidant properties}

DPPH scavenging assay was used to calculate the total antioxidant content of the extract and AgNPs. In a typical procedure, $100 \mu \mathrm{L}$ of DPPH solution $(0.25 \mathrm{mM}$ in methanol) was transferred to each well of a 96-well plate. Then, different concentrations of CSN, extract, and AgNPs $(5-400 \mu \mathrm{g} / \mathrm{mL})$ were added to each well. The plate was incubated in dark for $1-2$ hour at $37^{\circ} \mathrm{C}$. UV absorbance (A) of the sample was then assessed at $517 \mathrm{~nm}$ using a UV-Vis spectrophotometer (Mettler Toledo V670). The percentage of inhibition was calculated according to Equation $1 .{ }^{23}$

$$
\% \text { inhibition }=\frac{\mathrm{A}_{\text {control }}-\mathrm{A}_{\text {test }}}{\mathrm{A}_{\text {control }}} \times 100
$$

In this equation, $\mathrm{A}_{\text {control }}$ represents the absorbance of the solution prepared by adding $100 \mu \mathrm{L}$ of deionized $\mathrm{H}_{2} \mathrm{O}$ to $100 \mu \mathrm{L}$ of $0.25 \mathrm{mM}$ methanolic solution of DPPH. $\mathrm{A}_{\text {test }}$ refers to the absorbance of the AgNPs, walnut green husk extract, or CSN.

\section{Cytotoxicity evaluation of AgNPs}

To determine the cytotoxic and anticancer effects of AgNPs, cell viability study was conducted using the conventional MTT reduction assay. ${ }^{24-26}$ Briefly, L-929 and MCF-7 cells were seeded in 96-well plates at the density of 5,000 cells/well in the presence of $100 \mu \mathrm{L}$ cell culture medium (RPMI supplemented with $10 \%$ FBS and $1 \%$ penicillin-streptomycin solution). Cells were incubated for 24 hours in an incubator containing $5 \% \mathrm{CO}_{2}$ at $37^{\circ} \mathrm{C}$. After 24 hours of seeding, the medium inside the wells was replaced with fresh medium along with different concentrations of the walnut green husk extract, CSN, or AgNPs $(10-60 \mu \mathrm{g} / \mathrm{mL})$ and incubated for $24-48$ hours at $37^{\circ} \mathrm{C}$. To detect cell viability, the old medium was replaced with $100 \mu \mathrm{L}$ of fresh medium, then $10 \mu \mathrm{L}$ of MTT solution ( $5 \mathrm{mg} / \mathrm{mL}$ in DMSO) was added to each well, and the plates were incubated further for another 4 hours. The MTT solution was then discarded, and $100 \mu \mathrm{L}$ of DMSO was added to each well followed by incubation for 40 minutes in dark. The solution was then pipetted and its absorbance was recorded at $492 \mathrm{~nm}$ using a microplate reader (Bio-Rad Laboratories, Inc., Hercules, CA, USA).

\section{Antimicrobial tests}

The antimicrobial activity of the AgNPs was investigated by agar well diffusion method against the nosocomial strains of E. coli, $P$. aeruginosa, and $S$. aureus, and the standard strains of the same bacteria for comparison. In a typical test, the $0.5 \mathrm{McF}$ arland standard suspensions of the bacterial strains were prepared using a UV-Vis spectrophotometer at $\mathrm{OD}_{600 \mathrm{~nm}}$. The bacterial strains were then swabbed onto Mueller-Hinton agar (MHA) plates, and wells were formed by punching the medium using a sterilized Pasteur pipette. Then $60 \mu \mathrm{L}$ of the AgNPs suspension was added to each well in concentrations from 16 to $1,000 \mu \mathrm{g} / \mathrm{mL}$ and the plates were incubated at $37^{\circ} \mathrm{C}$ for 18 hours. The diameter of the inhibition zone was measured in $\mathrm{mm}$. Minimum inhibitory concentration (MIC) and minimum bactericidal concentration (MBC) were evaluated using the standard broth dilution method. ${ }^{27}$ Briefly, $50 \mu \mathrm{L}$ of the overnight-grown bacterial cultures $(0.01$ of $0.5 \mathrm{McF}$ arland) along with $100 \mu \mathrm{L}$ uncultured MHA were placed into 96-well plates followed by addition of $100 \mu \mathrm{L}$ of the AgNPs by serial dilutions of $5-100 \mu \mathrm{g} / \mathrm{mL}$ and incubated at $37^{\circ} \mathrm{C}$ for 18 hours. Wells containing culture media and the 
bacterial suspension were considered as control. The inhibition rate of the nanoparticles was measured spectrometrically at $\mathrm{OD}_{600 \mathrm{~nm}}$. To confirm the bacterial death, the wells showing no visible growth (more than MIC) were swabbed on MHA plates and incubated for 18 hours at $37^{\circ} \mathrm{C}$. The $\mathrm{MBC}$ is the lowest broth dilution of the antimicrobial that prevents growth of the organism on the agar plate. ${ }^{28}$

\section{Results and discussion}

\section{UV spectroscopic analysis of AgNPs}

The process of AgNP synthesis was monitored by the change of color, $\mathrm{pH}$, and $\mathrm{UV}-\mathrm{V}$ is spectra. Figure 1 shows 1) the UV-Vis spectra and 2) $\mathrm{pH}$ change of the colloidal AgNPs obtained using walnut green husk extract as a function of reaction time. The color of mixtures was changed from light brown to dark brown after addition of the extract from walnut green husk (Figure 1C) during the 24-hour period. According to the results, increasing the time of the reaction up to 24 hours results in a steady increase in the intensity of the absorbance peaks. These changes were accompanied by a red shift in $\lambda \max$ from 417 to $424 \mathrm{~nm}$ for the AgNPs. It is well known that AgNPs exhibit red-brown color in water, which is due to the excitation of surface plasmon resonances of the metal nanoparticles. ${ }^{29}$ Increase in the intensity of the absorbance peak has already been reported by Shankar et al as a function of time, although no red shift was reported in their research. ${ }^{30}$ The red shift is attributed to the initiation of the growth phase of nanocrystals resulting in the synthesis of larger particles without aggregation, ${ }^{31-33}$ or the presence of extract constituents as a capping agent. ${ }^{34}$ However, it may alternatively be due to the change in the $\mathrm{pH}$ of the mixture occurred during participation of $\mathrm{OH}$ groups during the formation of AgNPs.

\section{X-ray powder diffraction}

XRD technique was employed in order to investigate the crystalline phase and the orientation of the synthesized nanoparticles. Based on the XRD spectra shown in Figure 2A, peaks at $2 \theta=38^{\circ}, 44^{\circ}, 44.56^{\circ}, 64^{\circ}$, and $77^{\circ}$ are attributed to $(111,200,220)$, and (311), planes of the face-centered cubic geometry of Ag nanocrystals (JCPDS NO 01-087-0597). Moreover, appearance of three assigned peaks could be

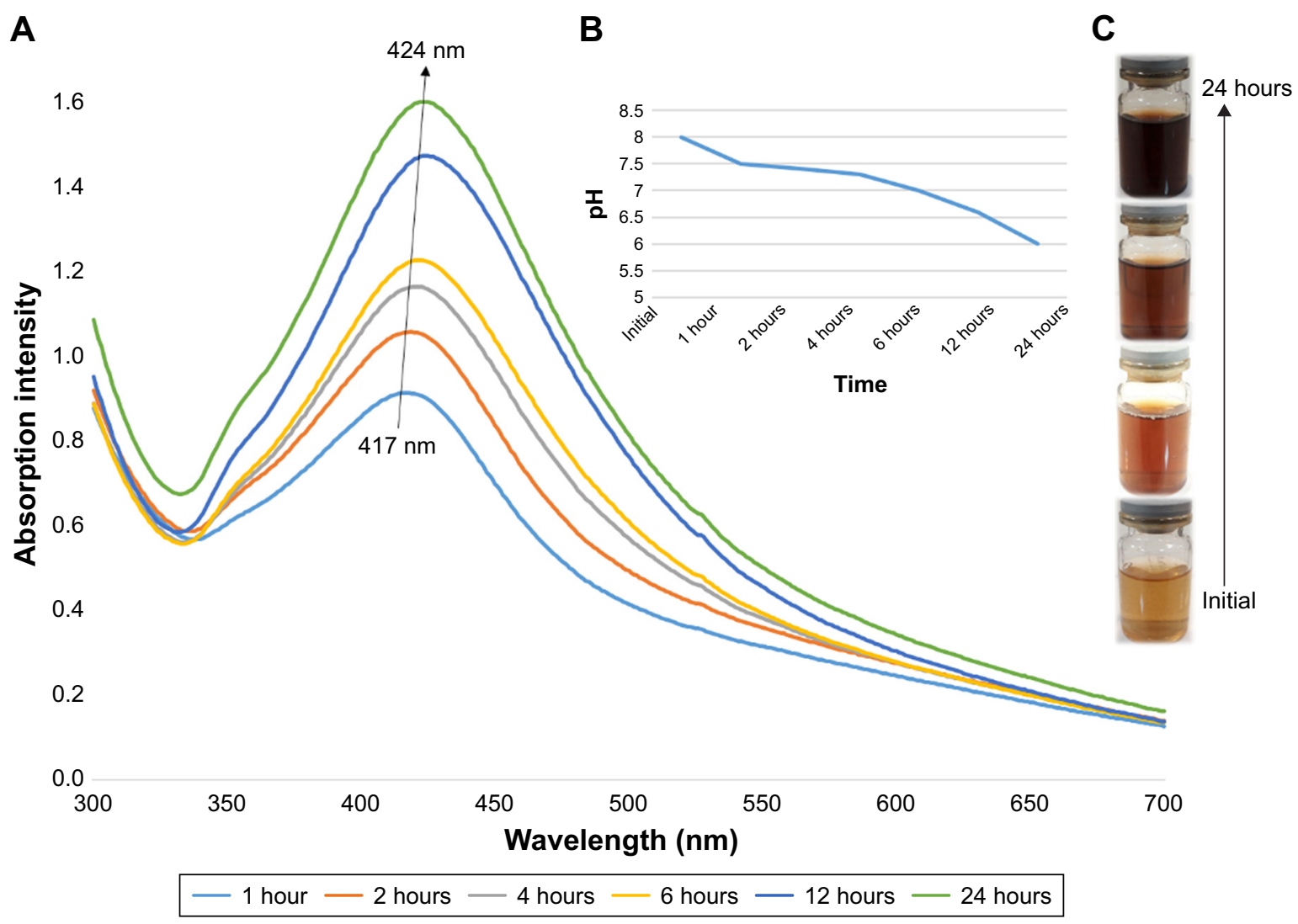

Figure I (A) UV-Vis spectra of colloidal AgNPs indicating increase in the peak intensity with time, along with a redshift. (B) $\mathrm{pH}$ value of the mixture gradually decreased during the reaction time. (C) Color change of the mixture from light yellow to dark brown during the reaction, which indicates synthesis of silver nanoparticles. Abbreviations: AgNPs, silver nanoparticles; UV-Vis, ultraviolet-visible. 

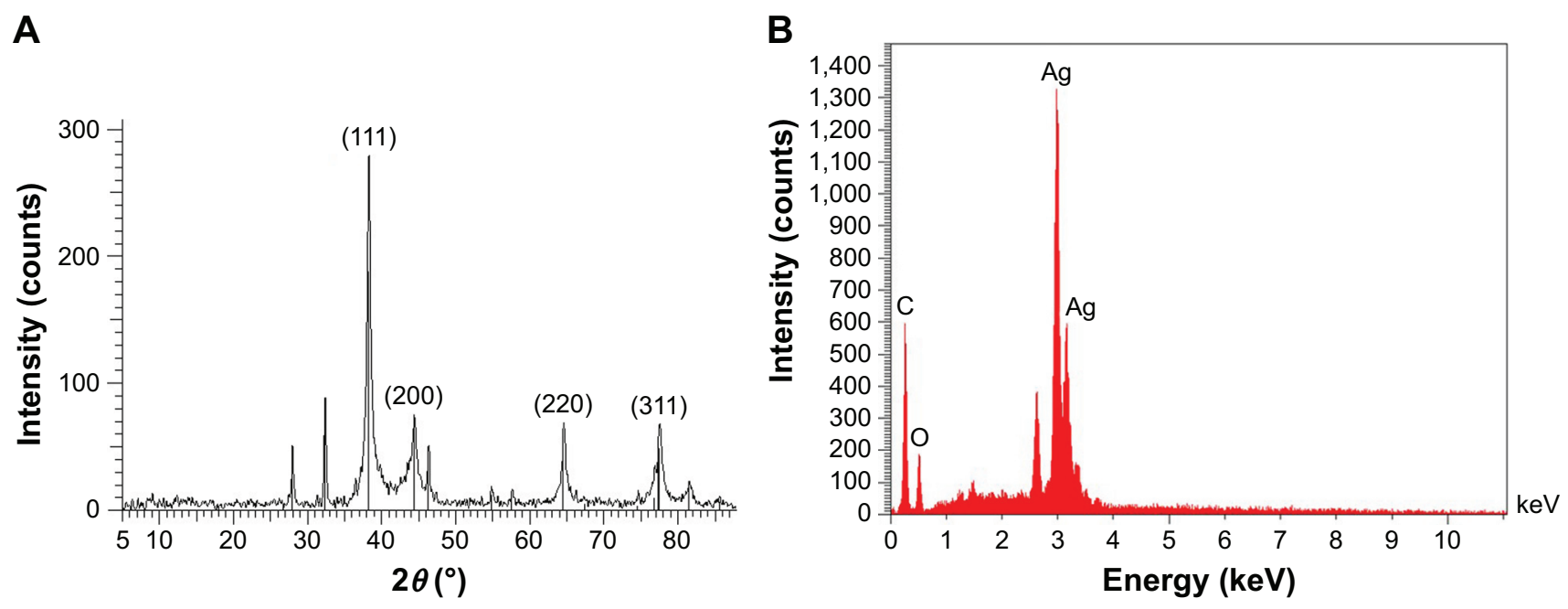

Figure 2 (A) X-ray powder diffraction spectrum of AgNPs. Peaks correspond to the face-centered cubic geometry of Ag crystal. (B) EDS spectrum of AgNPs. Presence of a sharp peak at $3 \mathrm{keV}$ confirms the presence of silver nanoparticles.

Abbreviations: AgNPs, silver nanoparticles; EDS, energy-dispersive X-ray spectroscopy.

interpreted as the presence of crystallized organic compounds on the nanoparticles. Besides, the EDS spectrum depicts a sharp peak at $3 \mathrm{keV}$ (Figure 2B), which confirms the XRD results.

\section{Particle characterization}

To investigate the hydrodynamic size, PDI, and surface zeta potential of the samples, the dynamic light-scattering (DLS) technique was utilized. According to the results shown in Figure 3A and B, the average hydrodynamic size, PDI, and zeta potential for AgNPs were $51 \mathrm{~nm}, 0.4$, and $-33.8 \mathrm{mV}\left(\mathrm{pH}=6.7,25^{\circ} \mathrm{C}\right)$, respectively. PDI can be defined as a number calculated from a two-parameter fit to the correlation data (the cumulants analysis). It is dimensionless and scaled such that the PDI value " 0 " represents monodisperse distribution whereas value "1" represents polydisperse distribution of particles. Values smaller

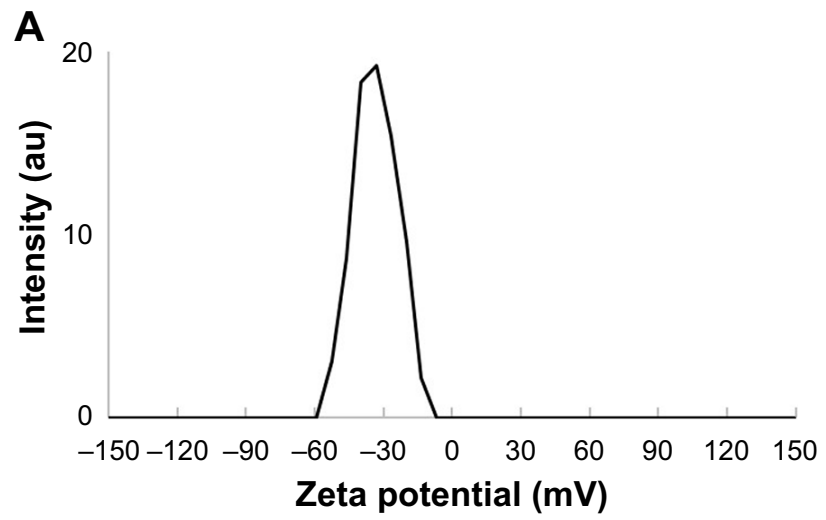

than 0.05 are mainly seen with highly monodisperse standards. PDI values bigger than 0.7 indicate that the sample has a very broad particle size distribution and is probably not suitable to be analyzed by the DLS technique. ${ }^{35,36}$ The PDI value of 0.4 obtained in this study is, hence, an acceptable value although there is room for improvements in this regard.

Zeta potential, on the other hand, is the measurement of the magnitude of electrostatic charge repulsion or attraction between particles in a liquid suspension. It is one of the essential parameters for characterizing the stability of nanoparticles in an aqueous environment. Particles with zeta potentials greater than $+30 \mathrm{mV}$ and less than $-30 \mathrm{mV}$ are considered stable for colloidal dispersions in the absence of steric stabilization. ${ }^{19,36}$ Consequently, the synthesized AgNPs with the zeta potential of $-33.8 \mathrm{mV}$ can be considered to be adequately stable colloidal system.

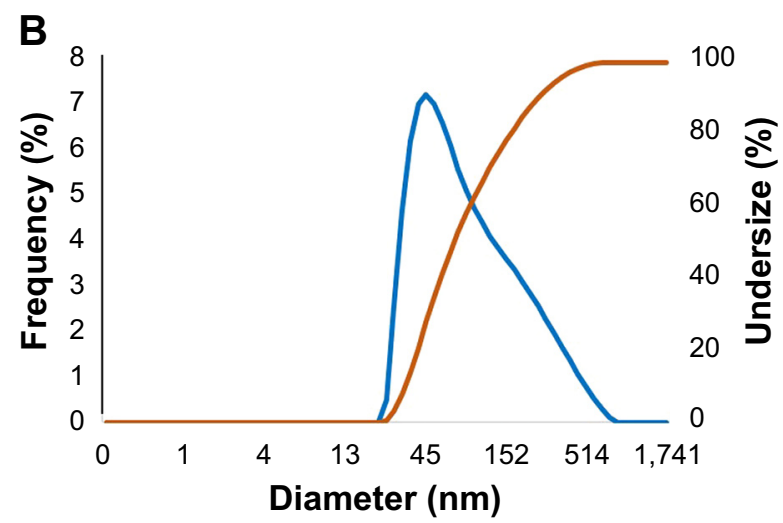

Figure 3 Dynamic light-scattering analysis of AgNPs indicating: (A) zeta potential and (B) mean diameter of the particles. Abbreviation: AgNPs, silver nanoparticles. 

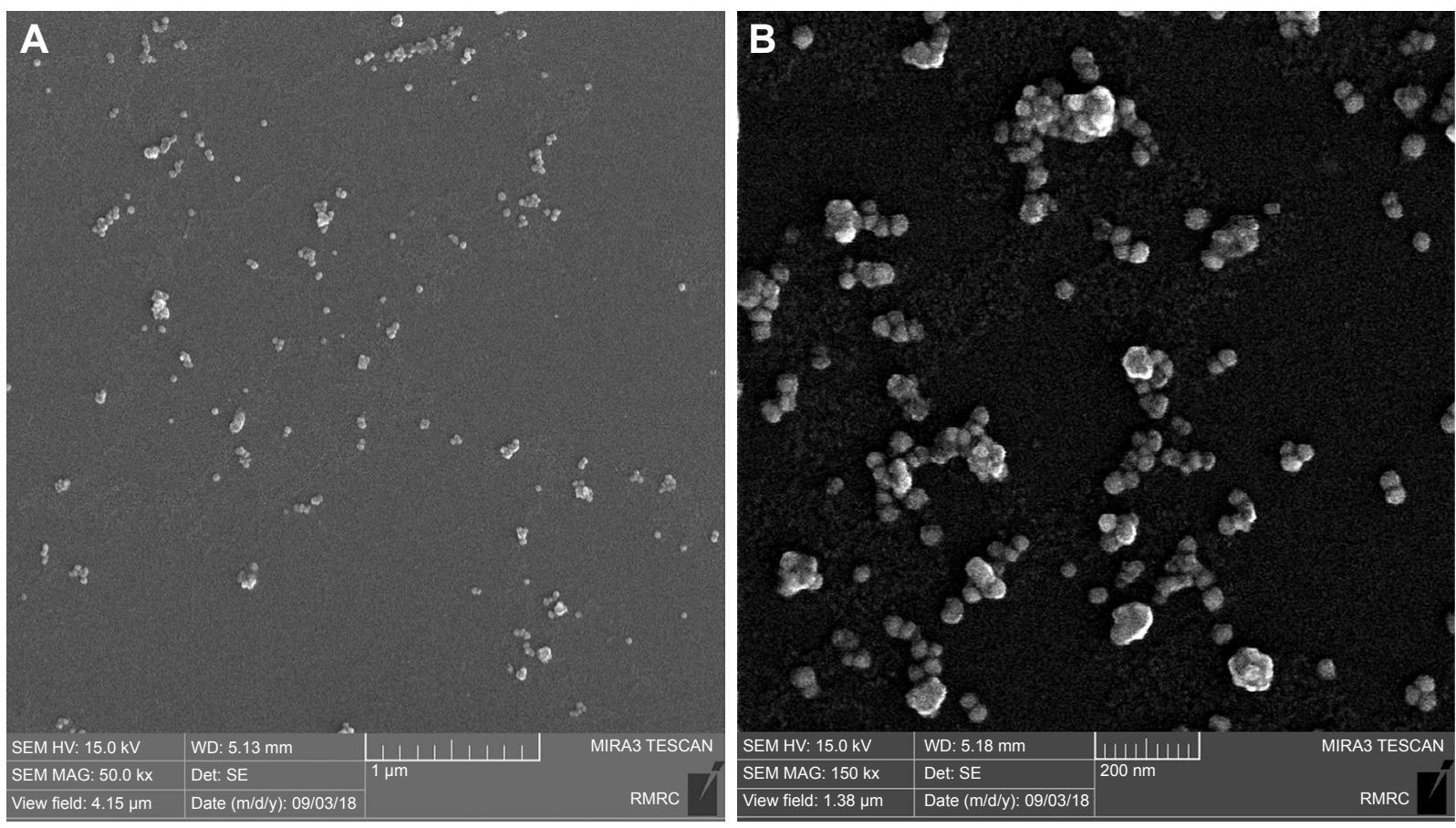

Figure 4 Representative field emission scanning electron microscopy (FESEM) imaging of green-synthesized silver nanoparticles.

Notes: (A) Scale bar: I $\mu \mathrm{m}$. (B) Scale bar: $200 \mathrm{~nm}$

Abbreviation: RMRC, Razi Metallurgical Research Center.

\section{Morphological analysis}

The FESEM micrographs shown in Figure 4 reveal that the nanoparticles possess spherical morphology, with an average size of $31.4 \mathrm{~nm}$. The difference between particle size data obtained by microscopic studies and DLS is due to the fact that a limited number of particles can be assessed by microscopic techniques (50 particles in this study) while DLS method measures all particles in the sample analyzed. The particle size distribution observed by the microscopic studies is relatively monodisperse, which is in accordance with the DLS data and the PDI value of 0.4. The clustering of the AgNPs seen in the Figure 4 is possibly a result of sample preparation procedure for the microscopic analysis. However, no distinct aggregation or agglomeration of the nanoparticles is expected basically due to the high electrostatic repulsive forces between the particles originated from their zeta potential value of $-33.8 \mathrm{mV}$ indicating stable colloidal system (as explained above). Moreover, no precipitation was observed in the synthesized AgNPs samples up to 6 months after their preparation and storage at $4^{\circ} \mathrm{C}$ as well as room temperature.

\section{FTIR}

To identify the chemical transformation that occurred during the interaction between the silver nitrate and functional groups present in green husk extract of walnut, FTIR analysis was carried out (Figure 5). Although the exact mechanism for the reduction of silver ions to AgNPs is not well understood, a simple comparison of FTIR spectra of the extract and the synthesized nanoparticles leads to the following extrapolation:

1. The extract spectrum matches well with the AgNPs spectrum (with a decrease in intensity and a slight shift in the position of peaks). These findings show that the functional groups such as $\mathrm{O}-\mathrm{H}\left(\right.$ at $\left.3,397 \mathrm{~cm}^{-1}\right)$ and $=\mathrm{C}-\mathrm{H}$ (at 2,925 $\mathrm{cm}^{-1}$ ) have a deterministic role in the reduction of silver ions. These functional groups are attributed to polyphenols and aldehydes as the main components of the walnut aqueous extract. ${ }^{37-41}$

2. There exists a decreasing and fractioning pattern for the peaks of functional groups such as $\mathrm{C}=\mathrm{O}\left(\right.$ at $\left.1,710 \mathrm{~cm}^{-1}\right)$, $\mathrm{N}-\mathrm{H}\left(\right.$ at $\left.1,603 \mathrm{~cm}^{-1}\right),-\mathrm{C}-\mathrm{H}\left(\right.$ at $1,401 \mathrm{~cm}^{-1}$ ), and $\mathrm{C}-\mathrm{O}$ (at $1,105 \mathrm{~cm}^{-1}$ ), which is the result of binding extract's components, including proteins and other organic molecules, on the surface of AgNPs. It is proposed that the proteins present in the walnut green husk extract function in a similar way to surfactant molecules by attaching to the nanoparticles surface and providing colloidal stability.$^{19}$ Moreover, it is proven that proteins can bind to the nanoparticles as the stabilizers either through their free amine groups or cysteine residues. ${ }^{42}$ Capping proteins and other organic compounds prevent agglomeration of nanoparticles in the suspension medium and are responsible for forming highly stable AgNPs. 

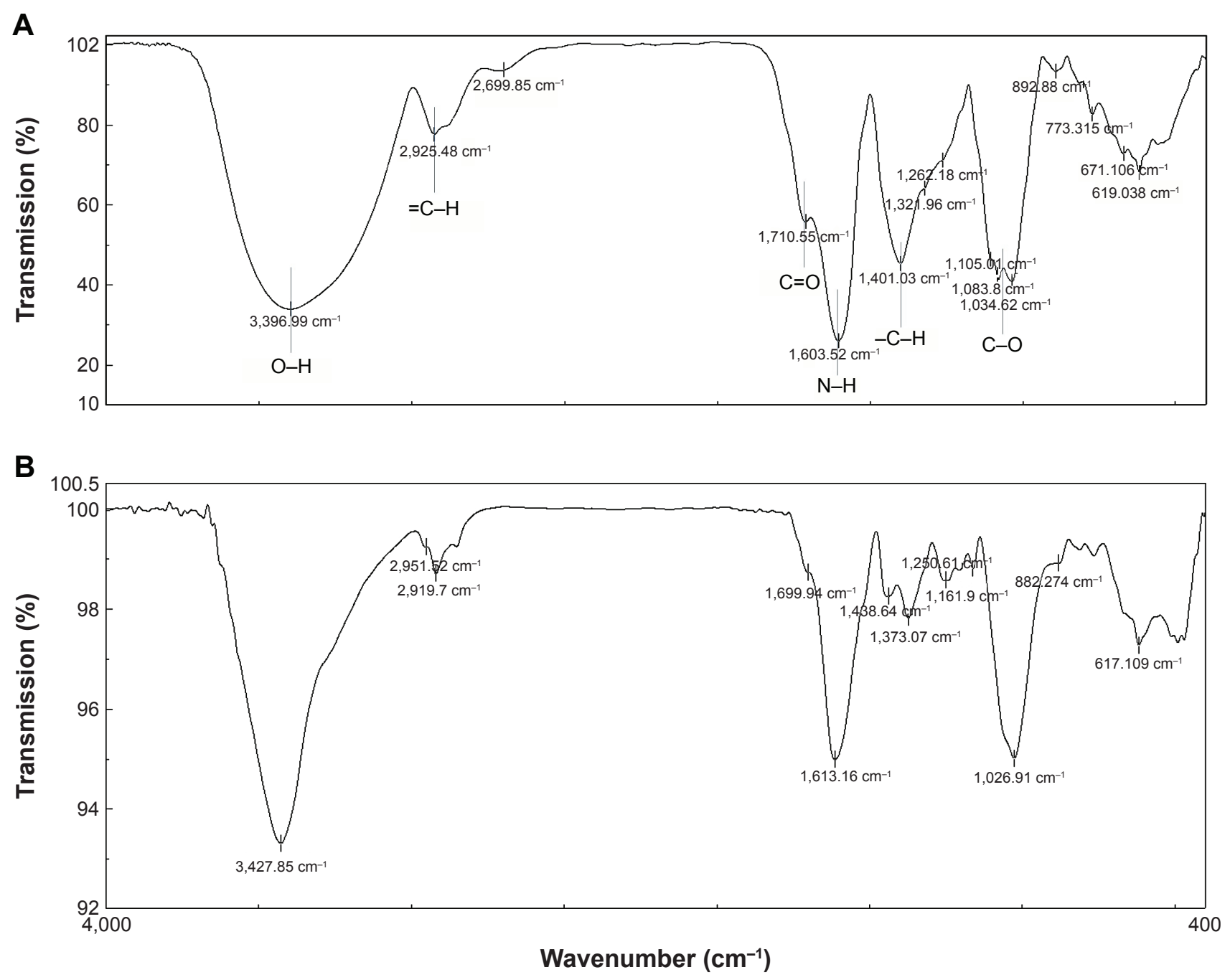

Figure 5 FTIR spectra of (A) walnut green husk extract and (B) AgNPs from 400 to $4,000 \mathrm{~cm}^{-1}$. The similarity of the two spectra confirms the synthesis of AgNPs using the extract.

Abbreviations: AgNPs, silver nanoparticles; FTIR, Fourier transform infrared.

\section{Antioxidant properties}

The results of DPPH-free radicals elimination studies (Figure 6) revealed that both aqueous extract of the walnut green husk and AgNPs are capable of scavenging elevated concentrations of the free radicals. CSN, however, exhibited less free-radical scavenging ability. The scavenging yields of CSN, extract, and AgNPs were calculated to be $34 \%, 45 \%$, and $78 \%$, respectively (after 2 hours exposure). The improved antioxidant property of the AgNPs seems to be dependent on the concentration and exposure time of the nanoparticles. As can be seen in Figure 6, in an exposure time of 1 hour, the $\mathrm{EC}_{50}$ of AgNPs decreased from 50 to $15 \mu \mathrm{g} / \mathrm{mL}$. In the case of walnut green husk extract, however, the antioxidant behavior was dependent only on the concentration of the extract. Although the ability of the extract to inhibit free radicals did not reach $50 \%$ in our experiments, a rational extrapolation predicts an $\mathrm{EC}_{50}=500 \mu \mathrm{g} / \mathrm{mL}$ for the walnut green husk extract. In a study using Portugal walnut green husk extract, Fernandez-Agullo et al reported an $\mathrm{EC}_{50}$ of $720 \mu \mathrm{g} / \mathrm{mL} .^{23}$ This value is less than the results obtained in the present study and indicates that the extract used is more potent in terms of free-radical scavenging compared to the previously reported findings. This elevated antioxidant activity could be attributed to the larger amount of biologically active compounds (polyphenols) such as flavonoids, ellagic acid, and valenic acid in the Persian walnut green husk compared to its Portuguese counterpart. ${ }^{43}$ It is proposed that the improved antioxidant property of nanoparticles is due to the simultaneous activity of polyphenols as antioxidant agents and AgNPs as a catalyst.

\section{Cytotoxicity}

The results of cytotoxicity investigations of different extract concentrations and AgNPs on L-929 fibroblast cells, as a normal cell line, and MCF-7, as a cancerous cell line, 


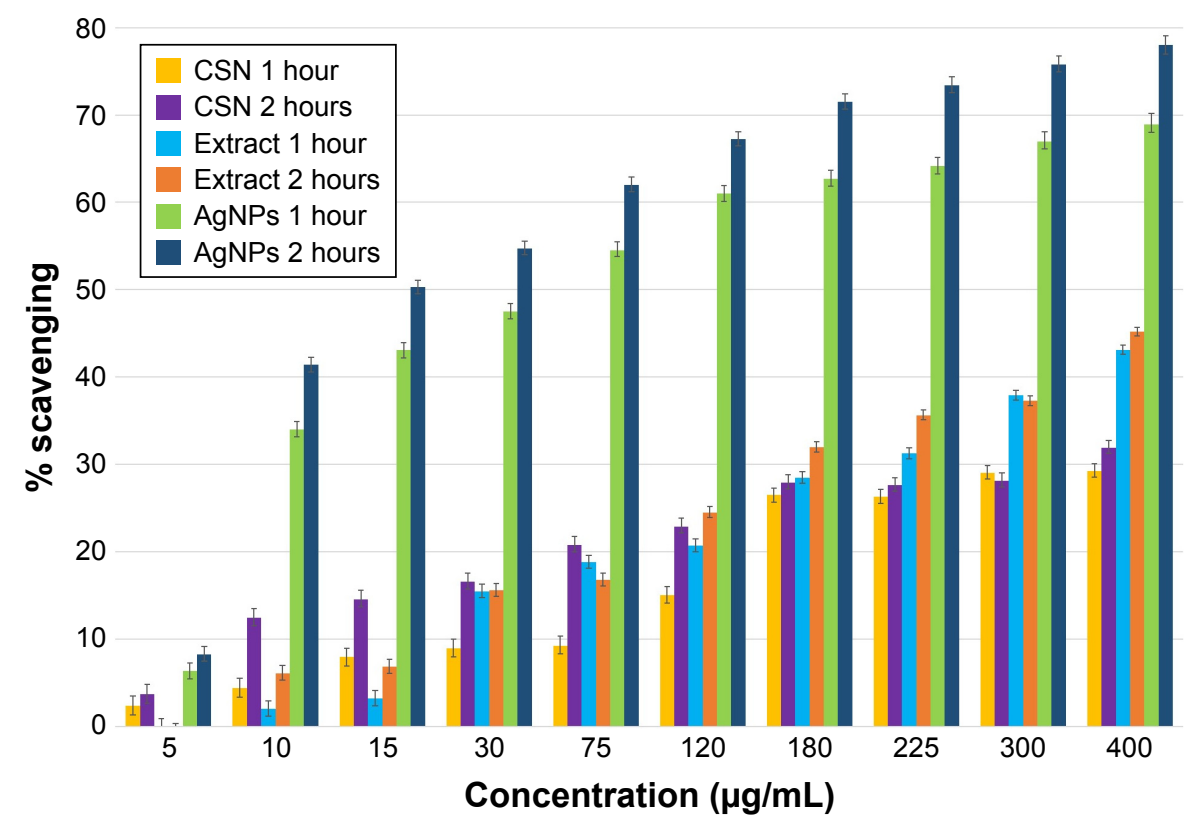

Figure 6 The antioxidant behavior of commercial silver nanoparticles (CSNs), walnut green husk extract, and AgNPs increasing in a concentration-dependent manner $(5-400 \mu \mathrm{g} / \mathrm{mL})$ and as a result of exposure time (I and 2 hours).

Abbreviation: AgNPs, silver nanoparticles.

revealed that both the extract and nanoparticles are cytotoxic against cancerous cell line while being nontoxic toward normal cell line. This is when the CSNs (employed as a control) exhibited cytotoxicity toward both tumor and normal cell lines (Figure 7C). The maximum cell death detected for the synthesized AgNPs was only $15 \%$ for the cultured L-929 cells after 48-hour exposure. This is when CSN resulted in $60 \%$ cytotoxicity toward the L-929 cells. However, the cytotoxicity of AgNPs and the extract against MCF-7 tumor cell line were $70 \%$ and $42 \%$, respectively (at the concentration of $60 \mu \mathrm{g} / \mathrm{mL}$ ). As can be seen in Figure 7, the cytotoxicity of AgNPs, CSN, and the extract are all concentration dependent.
Till now, several studies have proven that polyphenol compounds (especially ellagic acid) are safe toward healthy cells while showing cytotoxicity against cancerous cells. ${ }^{44-48}$ As previously mentioned, the walnut green husk is a rich source of polyphenols such as ellagic acid, which is responsible for the anticancer feature of the extract observed in this study. On the other hand, AgNPs with elevated activity due to their large surface to volume ratio could easily enter the cells, interact with cell constituents, and thus disturb the cellular signaling pathways. It has been postulated that AgNPs interact with mitochondria and disrupt the cellular electron transfer chain function leading to an increase in the ROS level. ${ }^{49-51}$ Consequently, the oxidative stress generated by ROS could be
A

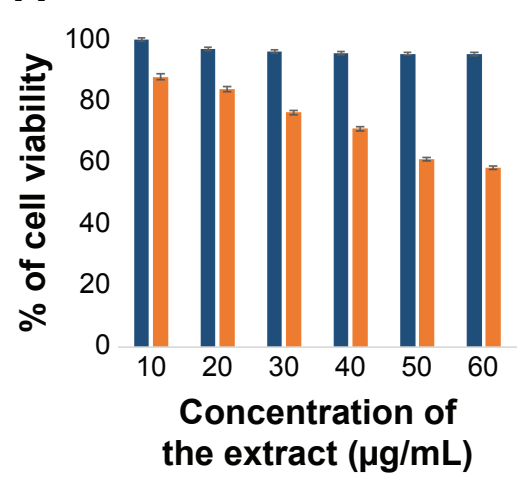

B

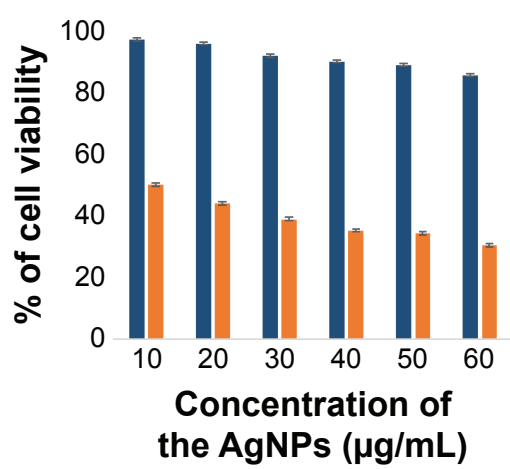

C

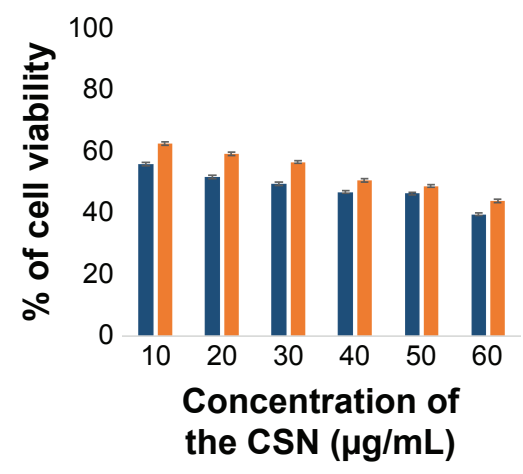

L-929 MCF-7

Figure 7 Cytotoxicity evaluation of (A) walnut green husk extract, (B) AgNPs, and (C) commercial silver nanoparticles (CSN) on L-929 and MCF-7 cell lines. Abbreviation: AgNPs, silver nanoparticles. 
Table I Inhibition zone diameter ( $\mathrm{mm}$ ), minimum inhibitory concentration (MIC), and minimum bactericidal concentration (MBC) of AgNPs against nosocomial (N.) and standard strains of different bacteria

\begin{tabular}{|c|c|c|c|c|c|c|c|c|}
\hline \multirow[t]{3}{*}{ Strains } & \multicolumn{6}{|c|}{ Diameter of inhibition zone $(\mathrm{mm})$} & \multirow{3}{*}{$\begin{array}{l}\text { MIC } \\
(\mu g / m L)\end{array}$} & \multirow{3}{*}{$\begin{array}{l}\text { MBC } \\
(\mu g / m L)\end{array}$} \\
\hline & \multicolumn{6}{|c|}{ AgNPs concentration $(\mu \mathrm{g} / \mathrm{mL})$} & & \\
\hline & 31 & 62 & 125 & 250 & 500 & 1,000 & & \\
\hline N. Staphylococcus aureus & - & - & - & - & - & 5 & 30 & 60 \\
\hline S. aureus II89 ATCC & - & - & - & - & - & 5 & 20 & 60 \\
\hline N. Pseudomonas aeruginosa & - & 7 & 14 & 16 & 20 & 20 & 20 & 20 \\
\hline P. aeruginosa 1214 PTCC & - & 10 & 13 & 15 & 16 & 18 & 10 & 20 \\
\hline N. Escherichia coli & - & 10 & 13 & 15 & 15 & 17 & 10 & 10 \\
\hline E. coli 35218 ATCC & - & 10 & 12 & 13 & 16 & 17 & 5 & 5 \\
\hline
\end{tabular}

Abbreviations: AgNPs, silver nanoparticles; ATCC, American Type Culture Collection; PTCC, Persian Type Culture Collection.

considered as a main toxicity mechanism of AgNPs against cells. The elevated anticancer activity of the AgNPs could be attributed to a synergy between AgNPs and the covering polyphenols. It is proposed that the superior cytotoxicity of AgNPs against cancerous cells occurs owing to the highest uptake of nanoparticles by these cells rather than healthy cells, given that cancerous cells have an abnormal metabolism and high proliferation rate, which in turn makes them more vulnerable. ${ }^{52}$ The simultaneous effect of AgNPs and polyphenols not only increases the ROS generation but also inhibits the transcription process. It is noteworthy that antioxidants such as polyphenols show cytotoxicity only against nonhealthy cells. ${ }^{43}$ This report is in good agreement with the data in the literature, which report the concentration-dependent toxicity of nanoparticles, particularly at lower levels. . $^{17,18,49}$

\section{Antimicrobial tests}

Different assessments such as well diffusion, standard broth dilution method, and assessment of turbidity of culture medium $\left(\mathrm{OD}_{600}\right)$ have been conducted to investigate the antimicrobial properties of the synthesized AgNPs. Based on the results presented in Table 1 and Figure 8, it is elucidated that these nanoparticles have significant antimicrobial behavior against the tested Gram-negative (E. coli and P. aeruginosa) and Gram-positive (S. aureus) bacteria.
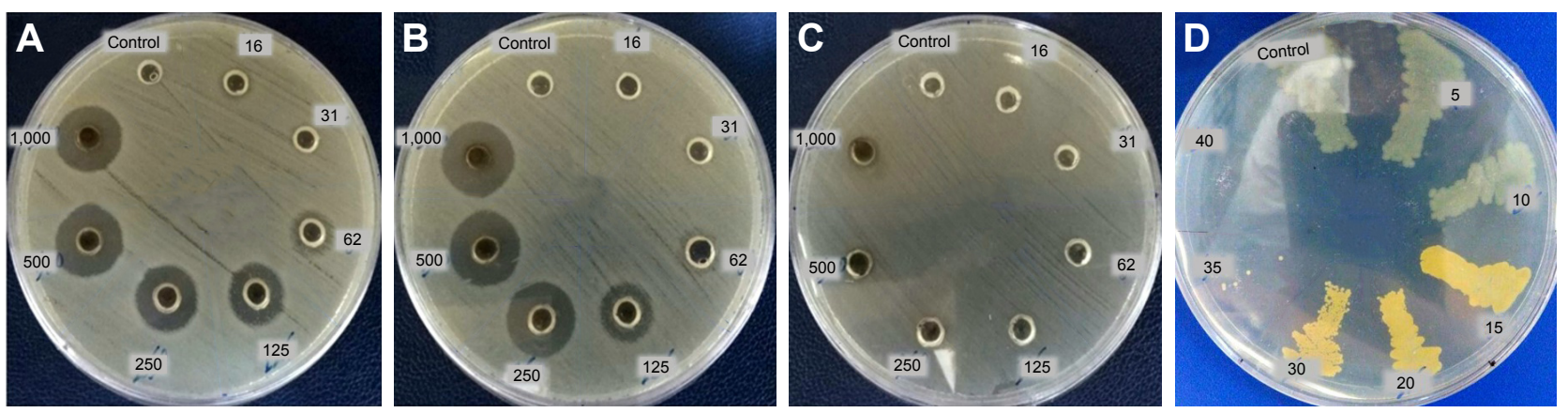

Figure 8 Antimicrobial efficacy of AgNPs against nosocomial strains of (A) Escherichia coli, (B) Pseudomonas aeruginosa, (C) Staphylococcus aureus, and (D) golden yellow colonies of $\mathrm{S}$. aureus strain after exposure to different concentrations of AgNPs. Abbreviation: AgNPs, silver nanoparticles. 


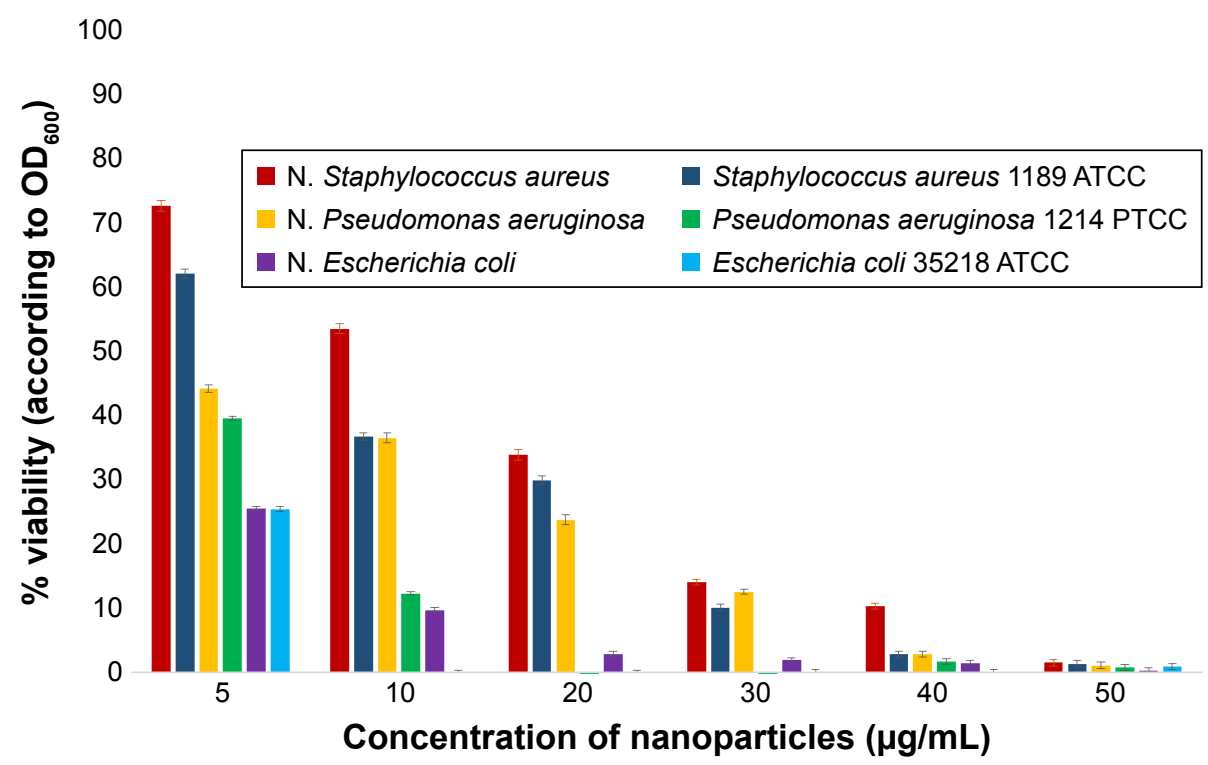

Figure 9 Microbial growth inhibition of AgNPs tested against nosocomial (N.) and standard bacterial strains. This graph shows that the AgNPs significantly inhibit bacterial growth even at low concentrations.

Abbreviation: AgNPs, silver nanoparticles.

that these nanoparticles can significantly decrease the microbial viability at concentrations lower than MIC (Figure 9).

The exact mechanism of antimicrobial activity of AgNPs is not known. However, the following four types of mechanisms have been proposed by researchers in this respect:

1. Interaction of AgNPs with cell membranes, alterations in the membrane permeability, and perturbation of respiratory chain enzymes.

2. Gradual diffusion of nanoparticles into the cells, which could both adversely affect the activity of cellular enzymes and restrict the transcription process by conjugation of silver particles to DNA.

3. Leakage of subcellular components as a result of nanoparticles interaction with the plasma membrane leading to cell death.

4. Generation of free radicals when the cell membrane is affected by silver ions. ${ }^{3,55-57}$

It seems that the prominent cell death mechanism is conjugation of nanoparticles with cells and change in the permeability of plasma membrane, which leads to free-radical and ROS generation. This assumption is further augmented by the emergence of pigments (such as beta carotene) in the tested bacteria as a defense mechanism against the oxidative stress as evident in Figure 8D and was also observed by other researchers. ${ }^{58,59}$ Same phenomena was also reported for copper oxide nanoparticles. ${ }^{60,61}$

It is worth mentioning that the antimicrobial activity of nanoparticles not only depends on their physicochemical characteristics but also depends on the biological features of target microorganisms. Results obtained in this study, which are in good agreement with other researcher's findings, demonstrated that the Gram-positive strains exhibited greater resistance against nanoparticles in comparison with Gram-negative ones. ${ }^{57,62,63}$ Herein, we have employed the same nanoparticles for both Gram-positive and Gramnegative bacterial strains, while obtaining different antimicrobial efficacies (Table 1). Results showed that the green synthesized AgNPs are able to kill E. coli when employed in a lower concentration than the concentrations used against $P$. aeruginosa and $S$. aureus. This observation can be explained by considering the differences between the cell wall of these three species. Presence of peptidoglycans in the structure of cell wall of $S$. aureus as well as its ability to produce pigments against ROS make it more resistant to the bactericidal effect of AgNPs.

\section{Conclusion}

Although the interest in the medical and nonmedical applications of metal nanoparticles is increasing, there are many remaining concerns regarding their safety and toxicity for humans as well as the environment. A very mild and solvent-free procedure was employed, in this study, at a low temperature for the extraction of herbal material from an agricultural waste. The walnut green husk extract was found to be highly capable of producing AgNPs with favorable physicochemical and biological properties. The synthesized AgNPs proved to possess improved anticancer, antioxidant, and antimicrobial activity in comparison with the extract and 
a CSN. The method of AgNP synthesis introduced in this study, therefore, holds great potential as a simple, low-cost, and environmentally friendly approach for producing valueadded products from waste material. The synthesized AgNPs exhibited selective cytotoxicity toward the cancerous cell line when compared to their effect on the normal cell line tested. These findings are very promising toward utilizing the biological effects of the AgNPs synthesized using walnut green husk extract.

\section{Disclosure}

The authors report no conflicts of interest in this work.

\section{References}

1. Zhou Y, Liang X, Dai Z. Porphyrin-loaded nanoparticles for cancer theranostics. Nanoscale. 2016;8(25):12394-12405.

2. Davies J, Davies D. Origins and evolution of antibiotic resistance. Microbiol Mol Biol Rev. 2010;74(3):417-433.

3. Rizzello L, Pompa PP. Nanosilver-based antibacterial drugs and devices: mechanisms, methodological drawbacks, and guidelines. Chem Soc Rev. 2014;43(5):1501-1518.

4. Loos C, Syrovets T, Musyanovych A, Mailänder V, Landfester K, Simmet T. Amino-functionalized nanoparticles as inhibitors of mTOR and inducers of cell cycle arrest in leukemia cells. Biomaterials. 2014; 35(6): 1944-1953.

5. Langer R, Tirrell DA. Designing materials for biology and medicine. Nature. 2004;428(6982):487-492

6. das SK, Khan MMR, Guha AK, Naskar N. Bio-inspired fabrication of silver nanoparticles on nanostructured silica: characterization and application as a highly efficient hydrogenation catalyst. Green Chem. 2013;15(9):2548.

7. Ahmed S, Annu, Chaudhry SA, Ikram S. A review on biogenic synthesis of $\mathrm{ZnO}$ nanoparticles using plant extracts and microbes: a prospect towards green chemistry. J Photochem Photobiol B. 2017;166: 272-284.

8. Adiseshaiah PP, Crist RM, Hook SS, Mcneil SE. Nanomedicine strategies to overcome the pathophysiological barriers of pancreatic cancer. Nat Rev Clin Oncol. 2016;13(12):750-765.

9. Enayati M, Nemati A, Zarrabi A, Shokrgozar MA. Reduced graphene oxide: an alternative for magnetic resonance imaging contrast agent. Mater Lett. 2018;233:363-366.

10. Luo C-H, Shanmugam V, Yeh C-S. Nanoparticle biosynthesis using unicellular and subcellular supports. NPG Asia Mater. 2015; 7(8):e209.

11. Rajan R, Chandran K, Harper SL, Yun S-I, Kalaichelvan PT. Plant extract synthesized silver nanoparticles: an ongoing source of novel biocompatible materials. Ind Crops Prod. 2015;70:356-373.

12. Patra JK, Baek K-H. Green Nanobiotechnology: factors affecting synthesis and characterization techniques. J Nanomater. 2014;2014(6): $1-12$.

13. Islami M, Zarrabi A, Tada S, Kawamoto M, Isoshima T, Ito Y. Controlled quercetin release from high-capacity-loading hyperbranched polyglycerol-functionalized graphene oxide. Int J Nanomedicine. 2018; 13:6059-6071.

14. Assadi Z, Emtiazi G, Zarrabi A. Novel synergistic activities of tetracycline copper oxide nanoparticles integrated into chitosan micro particles for delivery against multiple drug resistant strains: generation of reactive oxygen species (ROS) and cell death. J Drug Deliv Sci Technol. 2018; 44:65-70.

15. Dhoondia ZH, Chakraborty H. Lactobacillus mediated synthesis of silver oxide nanoparticles. Nanomater Nanotechnol. 2012;2(1):15.
16. Daniel SCGK, Banu BN, Harshiny M, et al. Ipomea carnea-based silver nanoparticle synthesis for antibacterial activity against selected human pathogens. J Exp Nanosci. 2014;9(2):197-209.

17. Dhand V, Soumya L, Bharadwaj S, Chakra S, Bhatt D, Sreedhar B. Green synthesis of silver nanoparticles using Coffea arabica seed extract and its antibacterial activity. Mater Sci Eng C Mater Biol Appl. 2016; 58:36-43.

18. Palaniappan P, Sathishkumar G, Sankar R. Fabrication of nano-silver particles using Cymodocea serrulata and its cytotoxicity effect against human lung cancer A549 cells line. Spectrochim Acta A Mol Biomol Spectrosc. 2015;138:885-890.

19. Nayak D, Ashe S, Rauta PR, Kumari M, Nayak B. Bark extract mediated green synthesis of silver nanoparticles: evaluation of antimicrobial activity and antiproliferative response against osteosarcoma. Mater $\mathrm{Sci}$ Eng C Mater Biol Appl. 2016;58:44-52.

20. Chanthini AB, Balasubramani G, Ramkumar R, et al. Structural characterization, antioxidant and in vitro cytotoxic properties of seagrass, Cymodocea serrulata (R. Br.) Asch. \& Magnus mediated silver nanoparticles. J Photochem Photobiol B Biol. 2015;153:145-152.

21. Mata R, Nakkala JR, Sadras SR, Colloids SSR. Biogenic silver nanoparticles from Abutilon indicum: their antioxidant, antibacterial and cytotoxic effects in vitro. Colloids Surf B Biointerf. 2015;128:276-286.

22. Patra S, Mukherjee S, Barui AK, Ganguly A, Sreedhar B, Patra CR. Green synthesis, characterization of gold and silver nanoparticles and their potential application for cancer therapeutics. Mater Sci Eng C Mater Biol Appl. 2015;53:298-309.

23. Fernández-Agulló A, Pereira E, Freire MS, et al. Influence of solvent on the antioxidant and antimicrobial properties of walnut (Juglans regia L.) green husk extracts. Ind Crops Prod. 2013;42(1):126-132.

24. Mostaghasi E, Zarepour A, Zarrabi A. Folic acid armed Fe3O4-HPG nanoparticles as a safe nano vehicle for biomedical theranostics. J Taiwan Inst Chem Eng. 2018;82:33-41.

25. Assadi Z, Emtiazi G, Zarrabi A. Hyperbranched polyglycerol coated on copper oxide nanoparticles as a novel core-shell nano-carrier hydrophilic drug delivery model. J Mol Liq. 2018;250:375-380.

26. Zarrabi A, Shokrgozar MA, Vossoughi M, Farokhi M. In vitro biocompatibility evaluations of hyperbranched polyglycerol hybrid nanostructure as a candidate for nanomedicine applications. $J$ Mater Sci Mater Med. 2014;25(2):499-506.

27. Balouiri M, Sadiki M, Ibnsouda SK. Methods for in vitro evaluating antimicrobial activity: a review. J Pharm Anal. 2016;6(2):71-79.

28. Sykes JE, Rankin SC. Isolation and identification of aerobic and anaerobic bacteria. In: Sykes J, editor. Canine and Feline Infectious Diseases. St. Louis: Elsevier Inc.; 2013:17-28.

29. Krishnaraj C, Jagan EG, Rajasekar S, Selvakumar P, Kalaichelvan PT, Mohan N. Synthesis of silver nanoparticles using Acalypha indica leaf extracts and its antibacterial activity against water borne pathogens. Colloids Surf B Biointerf. 2010;76(1):50-56.

30. Shankar SS, Ahmad A, Sastry M. Geranium leaf assisted biosynthesis of silver nanoparticles. Biotechnol Prog. 2003;19(6):1627-1631.

31. Arya G, Sharma N, Ahmed J, et al. Degradation of anthropogenic pollutant and organic dyes by biosynthesized silver nano-catalyst from Cicer arietinum leaves. J Photochem Photobiol B. 2017;174(April): 90-96.

32. Dong C, Cao C, Zhang X, et al. Wolfberry fruit (Lycium barbarum) extract mediated novel route for the green synthesis of silver nanoparticles. Optik - Int J Light Electron Optics. 2017;130:162-170.

33. Mukherjee P, Roy M, Mandal BP, et al. Green synthesis of highly stabilized nanocrystalline silver particles by a non-pathogenic and agriculturally important fungus T. asperellum. Nanotechnology. 2008; 19(7):075103

34. Vigneshwaran N, Nachane RP, Balasubramanya RH, Varadarajan PV. A novel one-pot "green" synthesis of stable silver nanoparticles using soluble starch. Carbohydr Res. 2006;341(12):2012-2018.

35. Danaei M, Dehghankhold M, Ataei S, et al. Impact of particle size and polydispersity index on the clinical applications of lipidic nanocarrier systems. Pharmaceutics. 2018;10(2):pii:E57. 
36. Bootz A, Vogel V, Schubert D, Kreuter J. Comparison of scanning electron microscopy, dynamic light scattering and analytical ultracentrifugation for the sizing of poly(butyl cyanoacrylate) nanoparticles. Eur J Pharm Biopharm. 2004;57(2):369-375.

37. Thakur N, Gaikar VG, Sen D, Mazumder S, Pandita NS. Phytosynthesis of silver nanoparticles using walnut (Juglans regia) bark with characterization of the antibacterial activity against Streptococcus mutans. Anal Lett. 2017;50(4):690-711.

38. Edison TJ, Sethuraman MG. Biogenic robust synthesis of silver nanoparticles using Punica granatum peel and its application as a green catalyst for the reduction of an anthropogenic pollutant 4-nitrophenol. Spectrochim Acta A Mol Biomol Spectrosc. 2013;104:262-264.

39. Oliveira I, Sousa A, Ferreira IC, Bento A, Estevinho L, Pereira JA. Total phenols, antioxidant potential and antimicrobial activity of walnut (Juglans regia L.) green husks. Food Chem Toxicol. 2008; 46(7):2326-2331.

40. Mystrioti C, Xanthopoulou TD, Tsakiridis P, Papassiopi N, Xenidis A. Comparative evaluation of five plant extracts and juices for nanoiron synthesis and application for hexavalent chromium reduction. Sci Total Environ. 2016;539:105-113.

41. Harshiny M, Matheswaran M, Arthanareeswaran G, Kumaran S, Rajasree S. Enhancement of antibacterial properties of silver nanoparticles-ceftriaxone conjugate through Mukia maderaspatana leaf extract mediated synthesis. Ecotoxicol Environ Saf. 2015;121:135-141.

42. Vinoj G, Pati R, Sonawane A, Vaseeharan B. In vitro cytotoxic effects of gold nanoparticles coated with functional acyl homoserine lactone lactonase protein from Bacillus licheniformis and their antibiofilm activity against Proteus species. Antimicrob Agents Chemother. 2015; 59(2):763-771.

43. Li L, Tsao R, Yang R, Liu C, Zhu H, Young JC. Polyphenolic profiles and antioxidant activities of heartnut (Juglans ailanthifolia Var. cordiformis) and Persian walnut (Juglans regia L.). J Agric Food Chem . 2006;54(21): 8033-8040.

44. Constantinou A, Stoner GD, Mehta R, Rao K, Runyan C, Moon R. The dietary anticancer agent ellagic acid is a potent inhibitor of DNA topoisomerases in vitro. Nutr Cancer. 1995;23(2):121-130.

45. Li TM, Chen GW, Su CC, et al. Ellagic acid induced p53/p21 expression, G1 arrest and apoptosis in human bladder cancer T24 cells. Anticancer Res. 2005;25(2A):971-980.

46. Dai J, Mumper RJ. Plant phenolics: extraction, analysis and their antioxidant and anticancer properties. Molecules. 2010;15(10):7313-7352.

47. Galati G, O’Brien PJ. Potential toxicity of flavonoids and other dietary phenolics: significance for their chemopreventive and anticancer properties. Free Radic Biol Med. 2004;37(3):287-303.
48. Madunić J, Madunić IV, Gajski G, Popić J, Garaj-Vrhovac V. Apigenin: a dietary flavonoid with diverse anticancer properties. Cancer Lett. 2018;413:11-22.

49. Park EJ, Yi J, Kim Y, Choi K, Park K. Silver nanoparticles induce cytotoxicity by a Trojan-horse type mechanism. Toxicol In Vitro. 2010; 24(3):872-878.

50. Bressan E, Ferroni L, Gardin C, et al. Silver nanoparticles and mitochondrial interaction. Int J Dent. 2013;2013:1-8.

51. Singh RP, Ramarao P. Cellular uptake, intracellular trafficking and cytotoxicity of silver nanoparticles. Toxicol Lett. 2012;213(2):249-259.

52. Cairns RA, Harris IS, Mak TW. Regulation of cancer cell metabolism. Nat Rev Cancer. 2011;11(2):85-95.

53. Lu L, Liu LJ, Chao WC, et al. Identification of an iridium(III) complex with anti-bacterial and anti-cancer activity. Sci Rep. 2015;5(1): 14544-14552.

54. Cheng G, Xu Y, Zhu X, et al. The antibacterial activities of aditoprim and its efficacy in the treatment of swine streptococcosis. Sci Rep. 2017; $7: 41370-41378$

55. Prabhu S, Poulose EK. Silver nanoparticles: mechanism of antimicrobial action, synthesis, medical applications, and toxicity effects. Int Nano Lett. 2012;2(1):32-41.

56. Taglietti A, Diaz Fernandez YA, Amato E, et al. Antibacterial activity of glutathione-coated silver nanoparticles against Gram positive and Gram negative bacteria. Langmuir. 2012;28(21):8140-8148.

57. Hajipour MJ, Fromm KM, Ashkarran AA, et al. Antibacterial properties of nanoparticles. Trends Biotechnol. 2012;30(10):499-511.

58. Liu GY, Essex A, Buchanan JT, et al. Staphylococcus aureus golden pigment impairs neutrophil killing and promotes virulence through its antioxidant activity. J Exp Med. 2005;202(2):209-215.

59. Diederich C, Leypold M, Culka M, et al. Mechanisms and specificity of phenazine biosynthesis protein PhzF. Sci Rep. 2017;7(1):6272-6284.

60. Applerot G, Lellouche J, Lipovsky A, et al. Understanding the antibacterial mechanism of $\mathrm{CuO}$ nanoparticles: revealing the route of induced oxidative stress. Small. 2012;8(21):3326-3337.

61. Kim S, Choi JE, Choi J, et al. Oxidative stress-dependent toxicity of silver nanoparticles in human hepatoma cells. Toxicol In Vitro. 2009; 23(6):1076-1084.

62. Guzman M, Dille J, Godet S. Synthesis and antibacterial activity of silver nanoparticles against Gram-positive and Gram-negative bacteria. Nanomed Nanotechnol Biol Med. 2012;8(1):37-45.

63. Shahverdi AR, Fakhimi A, Shahverdi HR, Minaian S. Synthesis and effect of silver nanoparticles on the antibacterial activity of different antibiotics against Staphylococcus aureus and Escherichia coli. Nanomed Nanotechnol Biol Med. 2007;3(2):168-171.
International Journal of Nanomedicine

\section{Publish your work in this journal}

The International Journal of Nanomedicine is an international, peerreviewed journal focusing on the application of nanotechnology in diagnostics, therapeutics, and drug delivery systems throughout the biomedical field. This journal is indexed on PubMed Central, MedLine, CAS, SciSearch ${ }^{\circledR}$, Current Contents ${ }^{\circledR} /$ Clinical Medicine,

\section{Dovepress}

Journal Citation Reports/Science Edition, EMBase, Scopus and the Elsevier Bibliographic databases. The manuscript management system is completely online and includes a very quick and fair peer-review system, which is all easy to use. Visit http://www.dovepress.com/ testimonials.php to read real quotes from published authors. 